\title{
Criteria of additivity in the protective effect of components of mixed corrosion inhibitors in chamber protection of zinc and steel
}

\author{
O.A. Betretdinova, A.Yu. Luchkin, O.A. Goncharova, Yu.I. Kuznetsov, \\ N.N. Andreev* and S.S. Vesely
}

A.N. Frumkin Institute of Physical Chemistry and Electrochemistry, Russian Academy of Sciences, Leninsky pr. 31, 119071 Moscow, Russian Federation

*E-mail: $\underline{n . a n d r e e v @ m a i l . r u}$

\begin{abstract}
The use of mixed formulations whose components complement and synergistically enhance the protective effect of each other is a promising method for increasing the efficiency of the inhibitory protection of metals. This article analyzes the criteria of additivity in the protective effect of components of mixed corrosion inhibitors. It is shown that they depend on the parameter used for estimating the protection efficiency. An equation is given that allows one to separate the effects of synergism and antagonism of inhibitors based on data on the times of complete metal protection by each of the mixture components, the mixture itself, and the corrosion resistance of the metal in the absence of inhibitors. A coefficient reflecting the mutual effect of the components of mixed inhibitors is introduced. The approach the we developed was used to estimate the mutual effect of components in mixtures of carboxylic acids (tridecanoic, stearic, oleic, linolenic) with urotropine in the chamber protection of zinc and steel. It has been shown that in the case of zinc, only oleic acid and urotropine synergistically enhance the protective effects of each other. The other mixtures are characterized by an antagonism in the protective effect. In contrast, in the case of steel, all the mixtures studied were characterized by well-pronounced synergism. Moreover, for the mixture based on stearic acid, the coefficient of mutual enhancement of the protective effect of components reaches 250. It has been found that the synergy in the protective effect is a direct indication that the mixed inhibitor should be used rather than any of its components. However, an antagonism is not a complete contraindication preventing the use of mixtures. For example, the antagonistic mixture of tridecanoic acid with urotropine protects zinc much more efficiently than its components taken separately. Taking the complexity of the chamber protection process into account, no unambiguous conclusion about the nature of antagonistic and synergistic interactions of components in mixed inhibitors are made. However, it is assumed that the interactions of compounds during their adsorption on metals are in question.
\end{abstract}

Keywords: corrosion inhibitors, mixed corrosion inhibitors, chamber metal protection, synergism of inhibitor action, criteria for the mutual effects of inhibitors.

Received: June 6, 2020. Published: June 10, 2020

doi: $\underline{10.17675 / 2305-6894-2020-9-2-24}$ 


\section{Introduction}

The use of inhibitors is a reliable and cost-effective method of corrosion protection in various fields of technology [1-5]. The range of inhibitors used in practice is very wide, but thousands of scientists around the world continue to work on the problem of creating more and more environmentally friendly and cheap formulations. The cost of inhibitory protection and the environmental burden created by it are directly related to the efficiency of inhibitors. The smaller the amount of inhibitors that provides the necessary level of metal protection, the more environmentally friendly and cost-effective their use is, other conditions being equal.

Traditionally, there are two ways for creating efficient inhibitors. The first one is associated with the search for or synthesis of new highly efficient individual compounds, while the second one involves the creation of inhibitor mixtures whose components complement and, in an ideal case, enhance the protective effects of each other. It should be noted that, as a rule, the preparation of mixed inhibitors is characterized by extreme simplicity of the production processes.

When the components of mixed inhibitors enhance the protective effect of each other, it is customary to call it synergistic interaction. The study of its mechanisms is one of the most urgent tasks in the modern inhibitor science. However, studies in this direction require a clear understanding of the laws of additivity, i.e., knowledge on what protective effect a mixed inhibitor should have in the absence of any interactions between its components.

Note that the laws of additivity are linked to specific criteria of the protection efficiency. They have been studied most thoroughly in relation to the coefficients of corrosion inhibition $(\gamma)$ in [6]. The conclusions of this work are directly based on the formal theory of the action of inhibitors [7]. Considering the low availability of source [6] the conclusions of which we will use below, let us re-derive the basic law of additivity suggested in it.

Let there be inhibitors 1 and 2 that are individually characterized by inhibition coefficients $\gamma_{1}$ and $\gamma_{2}$, respectively, in a corrosive medium of interest. Let the rate of general corrosion in this medium in the absence of inhibitors be $K_{0}$. Addition of inhibitor 1 to it will result in corrosion inhibition by a factor of $\gamma_{1}$ to give a value of $K_{1}$ :

$$
K_{1}=\frac{K_{0}}{\gamma_{1}}
$$

If $\gamma_{2}$ does not depend on the presence of inhibitor 1 in the system, i.e., in the absence of any interactions of the inhibitors, addition of inhibitor 2 to the system will further reduce the corrosion rate by a factor of $\gamma_{2}$. Then the corrosion rate in the system in the presence of both inhibitors 1 and $2\left(K_{\Sigma}\right)$ will be:

$$
K_{\Sigma}=\frac{K_{1}}{\gamma_{2}}
$$


Let us substitute $K_{1}$ from Equation 1 in this expression and get the following for the binary mixture:

$$
K_{\Sigma}=\frac{K_{0}}{\gamma_{1} \gamma_{2}}
$$

It follows from Equation 3 that:

$$
\frac{K_{0}}{K_{\Sigma}}=\gamma_{1} \gamma_{2}
$$

or, taking into consideration that $K_{0} / K_{\Sigma}=\gamma_{\Sigma}$ :

$$
\gamma_{\Sigma}=\gamma_{1} \cdot \gamma_{2}
$$

Equation 5 is a diagnostic criterion for the mutual effect of metal corrosion inhibitors. It was obtained for acid corrosion but also found use for neutral environments [8]. If $\gamma_{\Sigma}>\gamma_{1} \gamma_{2}$, then synergism is observed, i.e., the ability of inhibitors 1 and 2 to enhance the protective effects of each other. In this case, it makes sense to analyze the reasons for this enhancement.

In contrast, $\gamma_{\Sigma}<\gamma_{1} \gamma_{2}$ is an indication of an antagonism and weakening of the protective effect.

However, the corrosion rate and the corrosion inhibition coefficient are far from always used as characteristics of the efficiency of inhibitors. The full protection period, i.e., the time before the first corrosion damage appears on the metal, is used instead very often, especially in the case of atmospheric corrosion. This parameter is widely used in field tests, tests in a humidity or salt spray chamber, and in many others. It should be noted that for mixed inhibitors whose efficiency is estimated in this way, the diagnostic criteria for the mutual effect of the mixture components have not been found and a synergism is stated if the mixture provides complete protection of a metal for a longer period than its components do. Such an intuitive approach to the mutual effect of inhibitors is practiced in the analysis of the protective properties of volatile or chamber inhibitors, passivating compounds, inhibited thin-film coatings, etc.

Meanwhile, the additivity conditions are also derived quite easily in the estimation of the protective properties of mixed inhibitors based on the periods of total protection of metals that they provide. Deriving the diagnostic criterion for this case and analyzing the regularities obtained in the chamber protection of zinc and steel by mixed inhibitors were the purposes of this work.

\section{Theoretical analysis of the problem}

So, let's consider a system where the time until the first corrosion damage on the metal, i.e., the full protection time $(\tau)$, is used as the criterion of inhibitor efficiency. Like in the case considered above, we will analyze the mutual effects of the components of a binary mixture of inhibitors 1 and 2. 
Let the first corrosion damage on the metal become visually noticeable upon transition of a metal with mass $M$ on area $S$ to an oxide-hydroxide form. Then the rate of the corrosion process that determines the appearance of the first corrosion damage to the metal in the absence of inhibitors can be expressed as:

$$
K_{0}=\frac{M}{S \tau_{0}}
$$

where $\tau_{0}$ is the time until the first corrosion damage appears on the metal. It should be noted that both general and local corrosion may be taken into consideration.

Similarly, for inhibitor 1 with the metal protection period of $\tau_{1}$, inhibitor 2 with the metal protection period of $\tau_{2}$, and the mixture of inhibitors 1 and $2\left(\tau_{\Sigma}\right)$ the following expressions are valid:

$$
\begin{gathered}
K_{1}=\frac{M}{S \tau_{1}} \\
K_{2}=\frac{M}{S \tau_{2}}
\end{gathered}
$$

and

$$
K_{\Sigma}=\frac{M}{S \tau_{\Sigma}}
$$

By definition, the coefficient of metal corrosion inhibition by inhibitor 1 can be expressed as:

$$
\gamma_{1}=\frac{K_{0}}{K_{1}}=\frac{\left[\frac{M}{S \tau_{0}}\right]}{\left[\frac{M}{S \tau_{1}}\right]}=\frac{\tau_{1}}{\tau_{0}}
$$

Similarly:

$$
\gamma_{2}=\frac{K_{0}}{K_{2}}=\frac{\left[\frac{M}{S \tau_{0}}\right]}{\left[\frac{M}{S \tau_{2}}\right]}=\frac{\tau_{2}}{\tau_{0}}
$$

and

$$
\gamma_{\Sigma}=\frac{K_{0}}{K_{\Sigma}}=\frac{\left[\frac{M}{S \tau_{0}}\right]}{\left[\frac{M}{S \tau_{\Sigma}}\right]}=\frac{\tau_{\Sigma}}{\tau_{0}}
$$


Let us substitute the expressions for $\gamma$ into Equation 5 and obtain the following expression for a mixture of inhibitors 1 and 2 that do not interact with each other:

$$
\frac{\tau_{\Sigma}}{\tau_{0}}=\frac{\tau_{1}}{\tau_{0}} \frac{\tau_{2}}{\tau_{0}}=\frac{\tau_{1} \tau_{2}}{\tau_{0}^{2}}
$$

or

$$
\tau_{\Sigma}=\frac{\tau_{1} \tau_{2}}{\tau_{0}}
$$

This expression determines the additivity of the protective properties of inhibitors 1 and 2 expressed through the full metal protection times.

The situation where $\tau_{\Sigma}>\tau_{1} \tau_{2} / \tau_{0}$ is indicative of synergy, i.e., components 1 and 2 strengthen the protective effects of each other, while $\tau_{\Sigma}<\tau_{1} \tau_{2} / \tau_{0}$ is indicative of antagonism, i.e., mutual weakening of the protective effects.

Let us consider, based on this criterion, the results of a study on the protective effect of some mixed chamber corrosion inhibitors of zinc and steel under periodic moisture condensation conditions.

It should be reminded that chamber protection is a type of vapor-phase inhibitor protection. It is based on the protective after-effect of the adsorption layers of low-volatile corrosion inhibitors formed upon short-term exposure of metal products in vapors of inhibitors at elevated temperature [9-15].

\section{Experimental}

In this work, we estimated the ability of carboxylic acids (tridecanoic, stearic, oleic, linolenic), urotropine, and mixtures thereof to provide chamber protection.

All the reagents used in this study were of "pure" grade.

During the experiments, we used samples of zinc Ts0 and low-carbon steel St3 with dimensions of $30 \times 50 \times 1 \mathrm{~mm}$, with holes for mounting in test cells and chambers. Before the treatment of specimens with vapors of chamber inhibitors, their working surfaces were cleaned with sandpapers of various grain sizes, degreased with acetone and dried. Subsequently, the samples were mounted in sealed $0.5 \mathrm{~L}$ glass vessels containing a weighed portion of an inhibitor $(0.5 \mathrm{~g})$. The vessels were placed in an oven heated to $120^{\circ} \mathrm{C}$. The metal was treated with inhibitor vapors for $1 \mathrm{~h}$. After exposure in the chamber, the vessels were removed, allowed to cool to room temperature and kept for one day. After that, the samples were removed and subjected to corrosion tests with intense moisture condensation.

To perform a test, the specimens were attached with nylon fibers to the lids of airtight glass cells. The volume of each cell was $600 \mathrm{ml}$. Hot water $\left(0.1 \mathrm{~L}, 50^{\circ} \mathrm{C}\right)$ was poured into each cell. Once every 24 hours, each cell was opened and the cooled water was replaced with hot water. The replacement of water was combined with a visual inspection of the specimens. During the first 10 hours of exposure, the specimens were inspected once every hour without 
opening the cells. The time until the first corrosion damage appeared on the samples was determined in the tests.

\section{Results and Discussion}

The results of corrosion experiments are given in Table 1 .

Table 1. The protective effect of chamber corrosion inhibitors on zinc and steel.

\begin{tabular}{ccc}
\hline Chamber inhibitor & \multicolumn{2}{c}{$\begin{array}{c}\text { Time (hours) until the first } \\
\text { corrosion damage appears on: }\end{array}$} \\
steel \\
\hline Tridecanoic acid & 1.0 & 1.0 \\
Stearic acid & 48.0 & 4.0 \\
Oleic acid & 24.0 & 1.0 \\
Linolenic acid & 12.0 & 4.0 \\
Urotropine & 12.0 & 3.0 \\
Tridecanoic acid with urotropine & 5.0 & 2.0 \\
Stearic acid with urotropine & 144.0 & 48.0 \\
Oleic acid with urotropine & 72.0 & 504.0 \\
Linolenic acid with urotropine & 264.0 & 672.0 \\
\end{tabular}

After hot water was added to a cell, its vapors began to condense intensely on the metal samples. This caused rapid initiation of corrosion of the metals. Already in one hour after the start of experiments, corrosion damage was visible on the surface of metals that were not subjected to chamber treatment. It looked like gray spots on zinc and as a red deposit on steel.

\section{Chamber protection of zinc}

All the carboxylic acids studied inhibited zinc corrosion to some extent. Saturated carboxylic acids of normal structure (tridecanoic and stearic) protected zinc from corrosion for 24 to 48 hours under the conditions of our tests. However, the length of the hydrocarbon radical did not have a noticeable effect on the efficiency of the inhibitors. The use of higher unsaturated carboxylic acids for vapor phase protection did not give a noticeable improvement in the protective properties. Oleic (one double bond) and linolenic (three double bonds) acids protected zinc even worse than saturated stearic acid.

Urotropine was markedly inferior to carboxylic acids in efficiency. The first corrosion damage on zinc treated in its hot vapors appeared 5 hours after the start of the experiment. 
Sufficiently high protective properties on zinc were demonstrated by mixtures of carboxylic acids with urotropine. For example, a mixture of tridecanoic acid with the latter totally protected zinc for 144 hours. However, analysis of the mutual effects of the components of this mixture indicates that no synergistic protective effects are observed. Indeed, $\tau_{1}$ (tridecanoic acid) is 48 hours in this case, $\tau_{2}$ (urotropine) is 5 hours, and $\tau_{0}$ (no inhibitor) is 1 hour. The calculation of $\tau_{\Sigma}$ by Equation 9 gives a value of 240 hours.

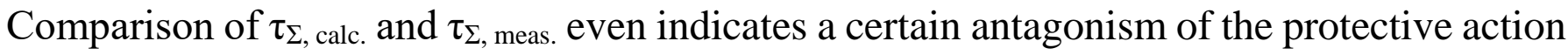
of the components.

The antagonistic effect is even more pronounced in the mixture of stearic acid and urotropine, which protects zinc relatively well ( $\tau_{\Sigma \text {, meas. }}=72$ hours). In this case, the value of

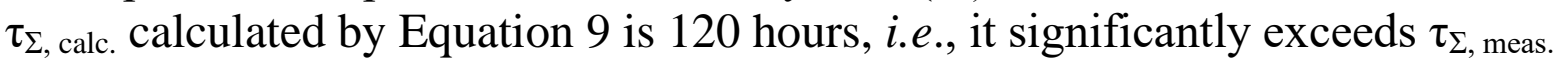

For convenience of evaluating the mutual effects of components in mixed inhibitors, let us introduce a special coefficient:

$$
\alpha=\frac{\tau_{\Sigma, \text { meas. }}}{\tau_{\Sigma, \text { calc. }}}
$$

Obviously, the value of $\alpha$ exceeding one indicates that there is a synergism in the protective effect. Moreover, the higher the $\alpha$ value, the more pronounced this interaction.

For the above mixtures of tridecanoic acid with urotropine and stearic acid with urotropine, the values of $\alpha$ were equal and amounted to 0.6 .

The mixture of oleic acid and urotropine, which protects zinc for 264 hours, is characterized by $\alpha=4.4$. Thus, we observe a weak synergism of the protective effect in the mixture in this case.

The mixture of linolenic acid with urotropine $\left(\tau_{\Sigma \text {, meas. }}=24\right.$ hours $)$ shows an antagonism of zinc protection. The value of $\alpha$ is as small as 0.4 in this case.

\section{Chamber protection of steel.}

The efficiency of all the individual compounds studied in the suppression of steel corrosion was significantly lower than on zinc.

The most efficient compounds studied, i.e., tridecanoic and oleic acids, protected the steel for no longer than 4 hours. Stearic acid did not show any protective properties. The time of total protection of the metal with linolenic acid was 3 hours. Urotropine provided steel protection for only 2 hours.

However, when carboxylic acids and urotropine were mixed, the situation changed dramatically. The components of these mixtures synergistically enhanced the protective effect of each other. The least efficient mixture, i.e., tridecanoic acid with urotropine, protected the metal for 48 hours and was characterized by $\alpha=6$. Mixtures of stearic or linolenic acids with urotropine protected the metal for the same quite long period of time, i.e., 504 hours. The coefficients $\alpha$ calculated for them were 252 and 84, respectively. The best protective properties (672 hours of total protection) were shown by the mixture of oleic 
acid with urotropine. It is noteworthy that the $\alpha$ value corresponding to this mixture was just a little above 80 , like in the case of the formulation based on linolenic acid.

To summarize the above information, it should be noted that the synergism of the protective effect is a direct indication that a mixed inhibitor should be used rather than any of its components. However, antagonism is not at all an indication that the mixtures must not be used. For example, the antagonistic mixture of tridecanoic acid with urotropine protects zinc much more efficiently than its components taken separately.

Chamber protection is rather a complex process for analysis of the mutual effects of the components of inhibitor mixtures. It implies the possibility of interaction of the components, both in the vapor phase and on the metal surface. It is difficult to make an unambiguous conclusion about the nature of these interactions based on the data presented. Nevertheless, significant differences in the coefficient of mutual effect of inhibitors for zinc and steel suggest that adsorption phenomena that depend on the nature of the adsorbent are concerned.

\section{Conclusions}

1. The use of mixed formulations whose components complement and synergistically enhance the protective effects of each other is a promising method for increasing the efficiency of the inhibitory protection of metals.

2. The criteria of the additivity in the protective effects of the components of mixed corrosion inhibitors, that is, their synergistic interaction or antagonism, depend on the parameter used for evaluating the protection efficiency. An equation is suggested that allows one to separate the effects of synergism and antagonism of inhibitors based on the times of total metal protection by each of the mixture components, the mixture itself, and the corrosion resistance of the metal in the absence of inhibitors. A coefficient reflecting the mutual effect of the components of mixed inhibitors is introduced.

3. As a rule, carboxylic acids and urotropine are antagonistic in the chamber protection of zinc. In the chamber protection of steel, the same components synergistically enhance each other's protective effects. It is assumed that these effects result from interactions of compounds during their adsorption on metals.

\section{References}

1. I.L. Rozenfel'd, Ingibitory korrozii (Corrosion inhibitors), Moscow, Khimiya, 1977 (in Russian).

2. V.P. Grigor'ev and V.V. Ekilik, Khimicheskaya struktura $i$ zashchitnoe deistvie ingibitorov korrozii (Chemical structure and protective effect of corrosion inhibitors), Rostov-on-Don, Rostov University Publishing House, 1978 (in Russian).

3. I.L. Rozenfeld and V.P. Persiantseva, Ingibitory atmosfernoi korrozii (Atmospheric corrosion inhibitors), Moscow, Nauka, 1985 (in Russian).

4. Yu.I. Kuznetsov, Organic inhibitors of corrosion of metals, New York-London, Plenum Press, 1996. 
5. V.S. Sastri, Corrosion inhibitors. Principle and applications, Wiley Chichester, New York, 1998.

6. V.V. Ekilik and O.V. Chikov, Diagnostic criteria of the mutual effect of inhibitors of acid corrosion of metals, Prot. Met., 1991, 27, no. 1, 59-68.

7. L.I. Antropov, E.M. Makushin and V.F. Panasenko, Ingibitory korrozii metallov (Metal corrosion inhibitors), Kiev, Tekhnika, 1981 (in Russian).

8. Y.I. Kuznetsov and N.N. Andreev, Mixed inhibitors and some aspects of synergism in corrosion inhibition, NACE International, Houston, TX (United States), 1996, CONF960389.

9. O.A. Goncharova, A.Yu. Luchkin, Yu.I. Kuznetsov, N.N. Andreev, N.P. Andreeva and S.S. Vesely, Octadecylamine, 1,2,3-benzotriazole and a mixture thereof as chamber inhibitors of steel corrosion, Int. J. Corros. Scale Inhib., 2018, 7, no. 2, 203-212. doi: 10.17675/2305-6894-2018-7-2-7

10. O.A. Goncharova, Yu.I. Kuznetsov, N.N. Andreev, A.Yu. Luchkin, N.P. Andreeva and D.S. Kuznetsov, A new corrosion inhibitor for zinc chamber treatment, Int. J. Corros. Scale Inhib., 2018, 7, no. 3, 340-351. doi: 10.17675/2305-6894-2018-7-3-5

11. O.A. Goncharova, A.Yu. Luchkin, N.N. Andreev, N.P. Andreeva and S.S. Vesely, Triazole derivatives as chamber inhibitors of copper corrosion, Int. J. Corros. Scale Inhib., 2018, 7, no. 4, 657-672. doi: 10.17675/2305-6894-2018-7-4-12

12. O.A. Goncharova, N.N. Andreev, A.Yu. Luchkin, Yu.I. Kuznetsov, N.P. Andreeva and S.S. Vesely, Protection of copper by treatment with hot vapours of octadecylamine, 1,2,3-benzotriazole and their mixtures, Mater. Corros., 2019, 70, no. 1, 161-168. doi: $10.1002 / \mathrm{maco} .201810366$

13. O.A. Goncharova, D.S. Kuznetsov, N.N. Andreev, Yu.I. Kuznetsov, N.P. Andreeva and S.S. Vesely, Corrosion screening of chamber inhibitors for an aluminum alloy, Int. J. Corros. Scale Inhib., 2019, 8, no. 2, 257-267. doi: 10.17675/2305-6894-2019-8-2-7

14. O.A. Goncharova, A.Yu. Luchkin, I.A. Archipushkin, N.N. Andreev, Yu.I. Kuznetsov and S.S. Vesely, Vapor-phase protection of steel by inhibitors based on salts of higher carboxylic acids, Int. J. Corros. Scale Inhib., 2019, 8, no. 3, 586-599. doi: 10.17675/2305-6894-2019-8-3-9

15. D.S. Kuznetsov, O.A. Goncharova, N.N. Andreev, N.P. Andreeva and S.S. Vesely, IFKhAN-140, a chamber corrosion inhibitor for brass, Int. J. Corros. Scale Inhib., 2020, 9, no. 1, 300-312. doi: 10.17675/2305-6894-2020-9-1-19 